\title{
Isolated Superior Mesenteric Artery Dissection: A Case Report and Literature Review
}

\author{
Hussein Daoud ${ }^{\mathrm{a}, \mathrm{c}}$, Ashraf Abugroun ${ }^{\mathrm{a}}$, Ahmed Subahi ${ }^{\mathrm{b}}$, Habeeb Khalaf ${ }^{\mathrm{a}}$
}

\begin{abstract}
Spontaneous isolated superior mesenteric artery dissection (ISMAD) is an uncommon cause of abdominal pain. Clinical presentation ranges from an asymptomatic incidental finding to acute bowel ischemia or fatal aneurysmal super mesenteric artery (SMA) rupture. We report the case of a 58-year-old male presenting with abdominal pain. Imaging studies revealed an ISMAD without radiological evidence of bowel ischemia. The patient was successfully treated using a conservative approach including bowel rest and anticoagulation. ISMAD incidence is expected to increase with the utilization of advanced imaging modalities. Thus, an ISMAD should be suspected when other common causes of an acute abdomen have been excluded. Given the lack of evidence-based guidelines, management options include conservative treatment and anticoagulation, endovascular stenting, or open surgical repair.
\end{abstract}

Keywords: Superior mesenteric artery dissection; Hypertension

\section{Introduction}

Spontaneous isolated superior mesenteric artery dissection (ISMAD) was first reported in 1947 by Bauersfeld [1]. It is an uncommon cause of abdominal pain, and it represents the most common type of mesenteric artery dissection. While an SMA dissection is commonly seen in association with aortic dissection, isolated lesions are a rare entity. Clinical presentation ranges from asymptomatic incidental findings to acute bowel ischemia or fatal aneurysmal SMA rupture. We report a case of a 58-year-old male who presented with abdominal pain. Imaging studies revealed an ISMAD without radiological evidence of bowel ischemia.

Manuscript submitted June 18, 2018, accepted August 8, 2018

${ }^{\text {aDepartment }}$ of Internal Medicine, Advocate Illinois Masonic Medical Center, 836 W Wellington Ave., Chicago, IL 60657, USA

bepartment of Internal Medicine, Detroit Medical Center/Wayne State University School of Medicine, Detroit, MI, USA

${ }^{\mathrm{c} C}$ Corresponding Author: Hussein Daoud, Department of Internal Medicine, Advocate Illinois Masonic Medical Center, 836 W Wellington Ave., Chicago, IL 60657, USA. Email: hussein.daoud@advocatehealth.com

doi: https://doi.org/10.14740/gr1056w

\section{Case Report}

A 58-year-old male with a past medical history of hypertension, hyperlipidemia, and tobacco use presented to the emergency department with abdominal pain localized to the epigastric and periumbilical areas. The pain was intermittent, not related to eating, and lasted for 3 days prior to admission. He had no fever, nausea, vomiting, or other gastrointestinal symptoms. The patient also denied any history of abdominal trauma or surgeries. On arrival, blood pressure was $156 / 104 \mathrm{~mm} \mathrm{Hg}$, and heart rate was 80 beats per minute (Fig. 1). His abdomen was soft, non-tender and non-distended, and with normal bowel sounds. The rest of the physical examination was unremarkable. The patient had a normal complete blood count (CBC), coagulation panel, and comprehensive metabolic panel (CMP). Lipid panel showed a total cholesterol level of $221 \mathrm{mg} / \mathrm{dL}$, low-density lipoprotein of $153 \mathrm{mg} / \mathrm{dL}$, high-density lipoprotein of $46 \mathrm{mg} / \mathrm{dL}$, and triglyceride of $110 \mathrm{mg} / \mathrm{dL}$. Computed tomography (CT) of the abdomen and pelvis with contrast revealed a short $(1.7 \mathrm{~cm})$ segment dissection of the superior mesenteric artery with mild induration of the adjacent fat (Fig. 2). The distal branches of the SMA appeared patent. The patient was treated conservatively with bowel rest, fluid resuscitation, intravenous heparin, and serial abdominal examinations. Home antihypertensive medications including daily amlodipine $5 \mathrm{mg}$, lisinopril $20 \mathrm{mg}$, and triamterene-hydrochlorothiazide 37.5-25 mg were resumed and his blood pressure was well controlled throughout admission (Fig. 1). The patient's condition improved and he was discharged on the third day of admission with a blood pressure of 103/70 $\mathrm{mm} \mathrm{Hg}$ and on warfarin with a target INR of $2-3$.

\section{Discussion}

ISMAD is usually seen in middle-aged males presenting with acute or chronic epigastric or upper left quadrant pain which can be related to meals. Other associated symptoms include nausea, vomiting, and diarrhea [2-4]. The predominance of diarrhea or malabsorption syndrome is commonly seen among patients who develop chronic bowel ischemia [2]. Physical examination findings include tenderness to palpation over the epigastric or left upper quadrant regions, and rarely, an audible epigastric bruit can be appreciated [5].

In a study by Yasuhara et al among 22 patients previously reported as having an ISMAD, the etiologic factors identified 


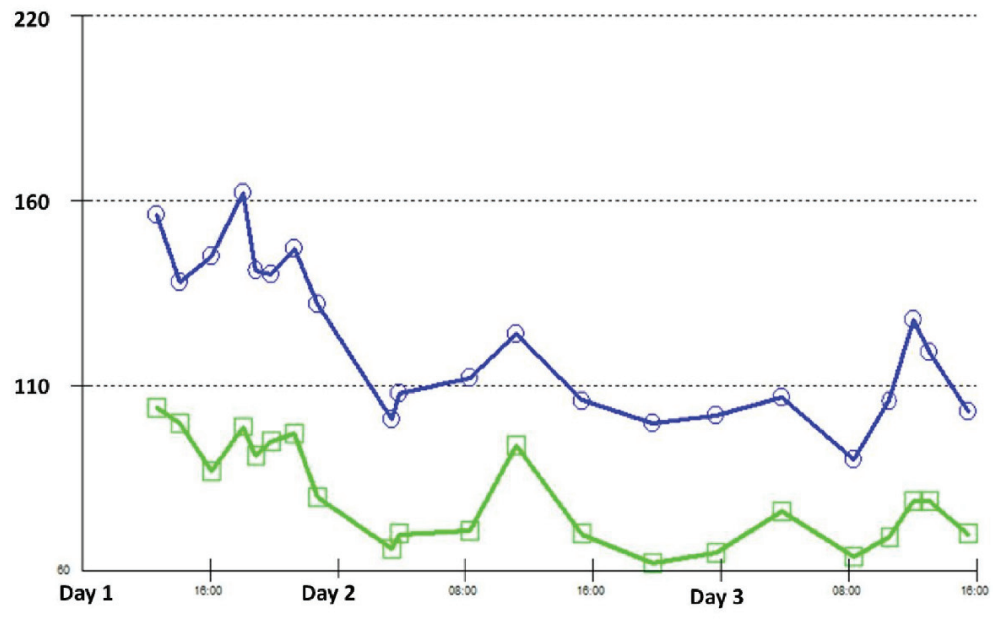

Figure 1. Systolic and diastolic blood pressure readings during hospitalization.

were atherosclerosis $(18 \%)$, medial necrosis or degeneration (14\%), fibromuscular dysplasia (5\%), and idiopathic (63\%) [6]. The prevalence of hypertension (HTN) among patients with an ISMAD is high, however, no direct cause-effect relationship with HTN was established [7]. The association between HTN and an ISMAD is far lower than in cases where a mesenteric artery dissection is found in conjunction with an aortic dissection. In a group of patients studied by Garrett and Park et al, $66 \%$ of patients with an aortic dissection had a history of HTN while only $31 \%$ of those with an ISMAD had HTN $[8,9]$.

The exact pathogenesis of an ISMAD remains uncertain, but it is observed that the dissection typically spares the origin of the super mesenteric artery, beginning 1.5 to $3 \mathrm{~cm}$ from the orifice of the SMA $[10,11]$. Solis et al proposed stress on the wall of the artery at the inferior pancreatic edge as a possible ISMAD trigger [12]. The transition at the lower margin of the pancreas, from a fixed to a relatively mobile state of the SMA, causes abnormal "shear stress" which can result in injury to the artery [13-15]. The previous speculation was supported by Park et al who proposed hemodynamic abnormalities in the SMA as a significant cause [8]. Park has observed from a review of CT angiography (CTA) scans in 51 patients that an ISMAD generally begins at the anterior wall of the SMA near the convex curvature [8]. With the use of a computer simulation model, abnormal hemodynamic factors were consistently observed at this site, suggesting mechanical stress as the etiology for SMA dissection [16].

Prior to 1975, when the first surgical repair was performed, an ISMAD was only diagnosed on postmortem examination [2]. Today, advanced imaging technology has improved the detection and diagnosis of an ISMAD. CT scans or magnetic resonance imaging are useful modalities for diagnosis. Detection of true and false lumens is perhaps the most characteristic sign of the existence of an ISMAD. This "double lumen sign" is often apparent on selective mesenteric angiography or CTA

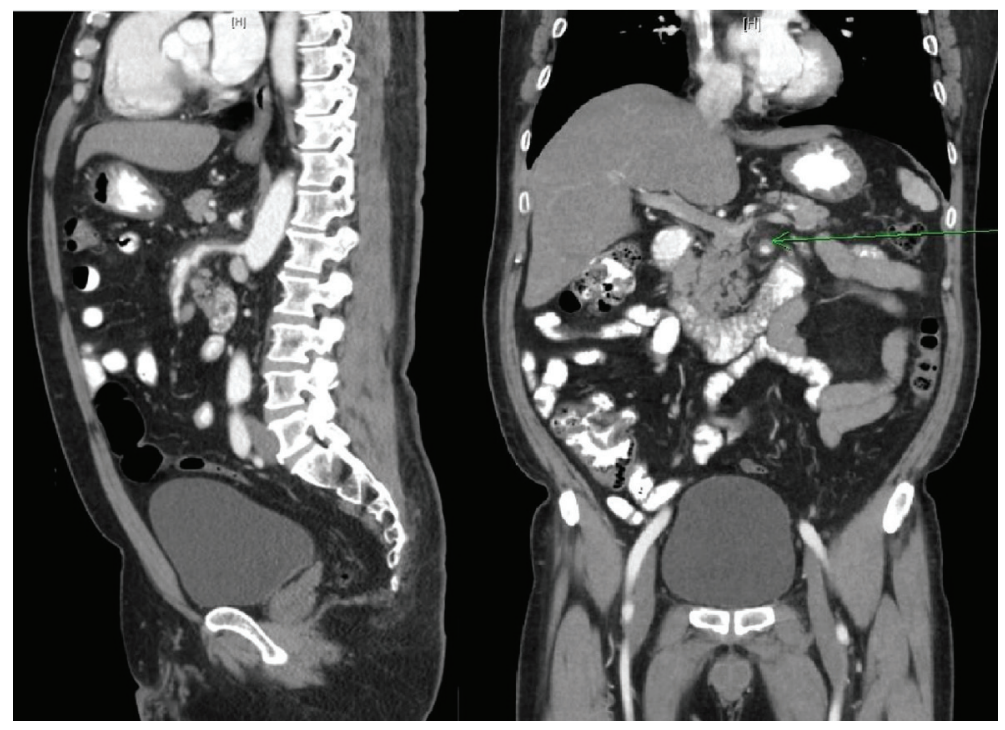

Figure 2. CT scan of the abdomen showing the area of SMA dissection and false lumen (arrow). 


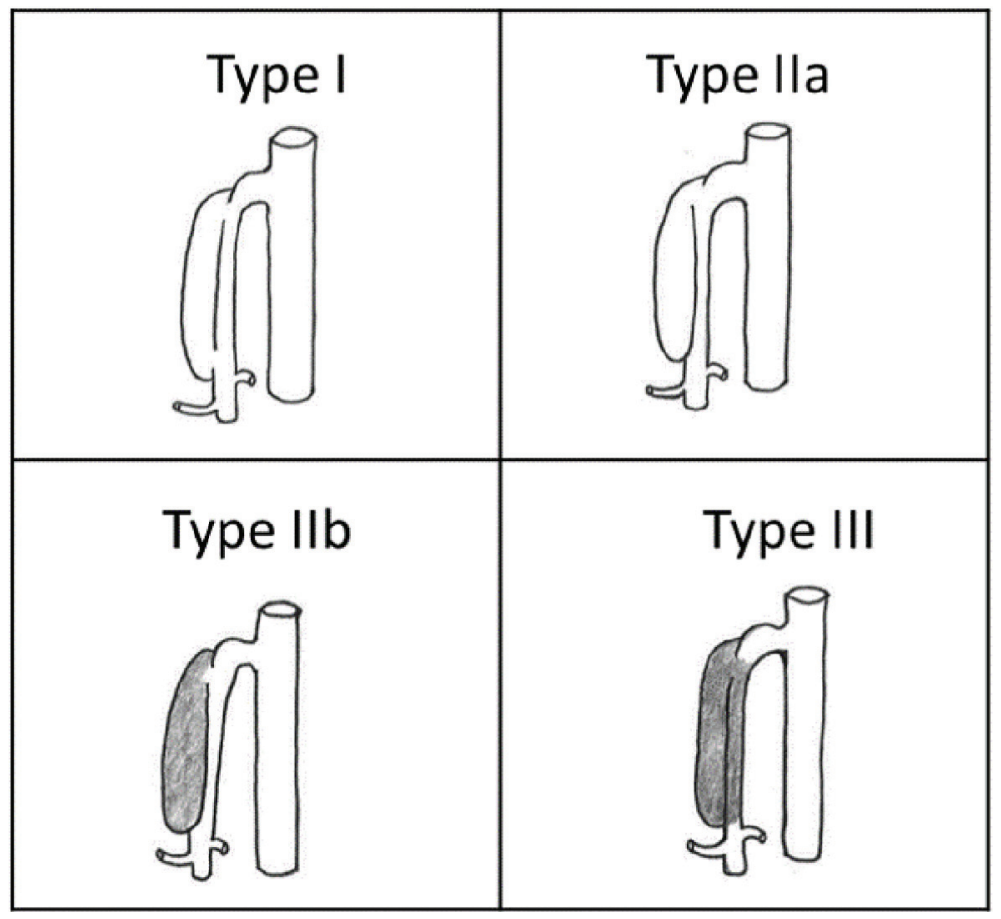

Figure 3. Angiographic characterization of ISMAD via cross-sectional and sagittal views of CT angiography proposed by Yun et al [20].

[13]. CTA is considered the gold standard for diagnosis as it allows the demonstration of the dissection flap, thrombosis, and dual lumen formation [17]. Increased fat attenuation in the area surrounding the SMA is a common radiological finding, and in cases where the intimal flap is not visible, increased SMA diameter with an increase in surrounding fat attenuation can be useful in diagnosis $[10,18,19]$. Furthermore, threedimensional CT scans can allow a better delineation of the anatomy and the distance between the SMA ostium and origin of the dissection.

Yun et al proposed a simplified categorization of an ISMAD based on CT angiogram assessment of dissection entry and re-entry points, length, patency, and degree of luminal stenosis at the dissected segment of the SMA [20]. These angiographic findings allowed categorization into three types depending on the presence of false luminal flow and luminal patency at the dissected segment (Fig. 3) [20]. Type I is characterized by a patent true lumen, false lumen, entry, and re-entry sites. Type II also features the bi-lumen structure, however, shows no re-entry flow from the false lumen. Type II is further classified into two types with type IIa exhibiting what Yun et al referred to as a "blind pouch of false lumen" due to the absence of any visible re-entry site. Type IIb is characterized by a thrombosed false lumen (i.e., no visible false luminal flow) causing narrowing in the true lumen. Type III features an SMA dissection with a clear blocking of the artery. Sakamoto et al also used a similar classification in their studies of ISMAD, where type II ISMAD was noted as having a cul-de-sac appearance of the false lumen [21].

Complications of an ISMAD include bowel infarction, acute peritonitis and pre-renal uremia as a late complication
$[15,22]$. Rupture of the SMA aneurysm or dissecting artery into the peritoneal cavity can result in intra-abdominal hemorrhage leading to hemorrhagic shock [23]. Thrombosis of the SMA with intestinal infarction has been recorded as a cause of death in four patients with an ISMAD, although all occurred prior to the advent of modern visceral artery reconstruction techniques [12]. Restenosis, thrombosis, and death related to chronic intestinal ischemia have also been reported after surgical revisualization and stenting [24].

There are no clear guidelines for the treatment of patients with an ISMAD. Available therapeutic options include conservative therapy, use of anticoagulation, and surgical intervention [2]. Generally, asymptomatic ISMAD patients or patients with a patent dual-lumen artery with no aneurysm formation respond best to medical therapy [11]. Among those who are treated medically, anticoagulation is commonly used $[2,25]$. Anticoagulation is thought to reduce the risk of thrombosis in the setting of exposed tissue factor. There is no agreement regarding the effectiveness of antiplatelet therapy when compared with anticoagulation $[19,26]$. On the other hand, surgical intervention is generally based on anatomic suitability, comorbidities, and symptom manifestation [11]. Surgical intervention with endovascular stent placement is indicated among patients with an ISMAD who are at risk for bowel ischemia or those who develop aneurysmal enlargement or rupture [11]. Zerbib et al proposed that patients with symptoms lasting greater than 7 days, minimum aneurysmal dilation of 2 $\mathrm{cm}$, and SMA stenosis are suitable surgical management candidates [24]. Compared to anticoagulation, endovascular stent placement results in a significant decrease in true lumen diameter [2]. Self-expanding stents are often recommended due to 
their weaker radial force [17].

\section{Conclusions}

ISMAD should be suspected when other common causes of acute abdominal pain have been excluded. Based on the clinical presentation, management options include conservative treatment and anticoagulation, endovascular stenting, or open surgical repair. Currently, there is no established guideline directed treatment algorithm. ISMAD detection is expected to increase with the utilization of advanced imaging including CTA and MRA, and this may lead to a reduction in complications.

\section{Acknowledgments}

Thanks to Dr. Vishnu Kuttappan from the radiology department for helping to interpret the images of the dissection and to Rayan Daoud for illustrating the different types of isolated superior mesenteric artery dissections.

\section{Conflict of Interest}

None of the authors have any conflict of interest to declare.

\section{Funding}

None of the authors have any source of funding to declare.

\section{References}

1. Bauersfeld SR. Dissecting aneurysm of the aorta; a presentation of 15 cases and a review of the recent literature. Ann Intern Med. 1947;26(6):873-889.

2. Gobble RM, Brill ER, Rockman CB, Hecht EM, Lamparello PJ, Jacobowitz GR, Maldonado TS. Endovascular treatment of spontaneous dissections of the superior mesenteric artery. J Vasc Surg. 2009;50(6):1326-1332.

3. Lalitha P, Reddy B. Isolated superior mesenteric artery dissection. Indian J Radiol Imaging. 2010;20(2):132-134.

4. Watring NJ, Smith CM, Stokes GK, Counselman FL. Spontaneous superior mesenteric artery (SMA) dissection: an unusual cause of abdominal pain. J Emerg Med. 2010;39(5):576-578.

5. Chang SH, Lien WC, Liu YP, Wang HP, Liu KL. Isolated superior mesenteric artery dissection in a patient without risk factors or aortic dissection. Am J Emerg Med. 2006;24(3):385-387.

6. Yasuhara H, Shigematsu H, Muto T. Self-limited spontaneous dissection of the main trunk of the superior mesenteric artery. J Vasc Surg. 1998;27(4):776-779.

7. DeCarlo C, Ganguli S, Borges JC, Schainfeld RM, Mintz AJ, Mintz J, Jaff MR, et al. Presentation, treatment, and outcomes in patients with spontaneous isolated celiac and superior mesenteric artery dissection. Vasc Med. 2017;22(6):505-511.

8. Park YJ, Park CW, Park KB, Roh YN, Kim DI, Kim YW. Inference from clinical and fluid dynamic studies about underlying cause of spontaneous isolated superior mesenteric artery dissection. J Vasc Surg. 2011;53(1):80-86.

9. Garrett HE, Jr. Options for treatment of spontaneous mesenteric artery dissection. J Vasc Surg. 2014;59(5):14331439 e $1431-1432$.

10. Suzuki S, Furui S, Kohtake H, Sakamoto T, Yamasaki M, Furukawa A, Murata K, et al. Isolated dissection of the superior mesenteric artery: CT findings in six cases. Abdom Imaging. 2004;29(2):153-157.

11. Sheldon PJ, Esther JB, Sheldon EL, Sparks SR, Brophy DP, Oglevie SB. Spontaneous dissection of the superior mesenteric artery. Cardiovasc Intervent Radiol. 2001;24(5):329-331.

12. Solis MM, Ranval TJ, McFarland DR, Eidt JF. Surgical treatment of superior mesenteric artery dissecting aneurysm and simultaneous celiac artery compression. Ann Vasc Surg. 1993;7(5):457-462.

13. Kim YW. Current Understandings of Spontaneous Isolated Superior Mesenteric Artery Dissection. Vasc Specialist Int. 2016;32(2):37-43.

14. Javerliat I, Becquemin JP, d'Audiffret A. Spontaneous isolated dissection of the superior mesenteric artery. Eur J Vasc Endovasc Surg. 2003;25(2):180-184.

15. Hirai $S$, Hamanaka $Y$, Mitsui N, Isaka M, Kobayashi T. Spontaneous and isolated dissection of the main trunk of the superior mesenteric artery. Ann Thorac Cardiovasc Surg. 2002;8(4):236-240.

16. Park YJ, Park KB, Kim DI, Do YS, Kim DK, Kim YW. Natural history of spontaneous isolated superior mesenteric artery dissection derived from follow-up after conservative treatment. J Vasc Surg. 2011;54(6):1727-1733.

17. Miyamoto N, Sakurai Y, Hirokami M, Takahashi K, Nishimori H, Tsuji K, Kang JH, et al. Endovascular stent placement for isolated spontaneous dissection of the superior mesenteric artery: report of a case. Radiat Med. 2005;23(7):520-524.

18. Okino Y, Kiyosue H, Mori H, Komatsu E, Matsumoto S, Yamada Y, Suzuki K, et al. Root of the small-bowel mesentery: correlative anatomy and CT features of pathologic conditions. Radiographics. 2001;21(6):1475-1490.

19. Han Y, Cho YP, Ko GY, Seo DW, Kim MJ, Kwon H, Kim $\mathrm{H}$, et al. Clinical outcomes of anticoagulation therapy in patients with symptomatic spontaneous isolated dissection of the superior mesenteric artery. Medicine (Baltimore). 2016;95(16):e3480.

20. Yun WS, Kim YW, Park KB, Cho SK, Do YS, Lee KB, Kim DI, et al. Clinical and angiographic follow-up of spontaneous isolated superior mesenteric artery dissection. Eur J Vasc Endovasc Surg. 2009;37(5):572-577.

21. Sakamoto I, Ogawa Y, Sueyoshi E, Fukui K, Murakami T, Uetani M. Imaging appearances and management of isolated spontaneous dissection of the superior mesenteric artery. Eur J Radiol. 2007;64(1):103-110.

22. Vignati PV, Welch JP, Ellison L, Cohen JL. Acute mes- 
enteric ischemia caused by isolated superior mesenteric artery dissection. J Vasc Surg. 1992;16(1):109-112.

23. Goueffic Y, Costargent A, Dupas B, Heymann MF, Chaillou P, Patra P. Superior mesenteric artery dissection: case report. J Vasc Surg. 2002;35(5):1003-1005.

24. Zerbib P, Lebuffe G, Sergent-Baudson G, Chamatan A, Massouille D, Lions C, Chambon JP. Endovascular versus open revascularization for chronic mesenteric ischemia: a comparative study. Langenbecks Arch Surg.
2008;393(6):865-870.

25. Ambo T, Noguchi Y, Iwasaki H, Kondo J, Matsumoto A, Suzuki H, Takamura Y. An isolated dissecting aneurysm of the superior mesenteric artery: report of a case. Surg Today. 1994;24(10):933-936.

26. Li Z, Ding H, Shan Z, Du J, Yao C, Chang G, Wang S. Initial and middle-term outcome of treatment for spontaneous isolated dissection of superior mesenteric artery. Medicine (Baltimore). 2015;94(45):e2058. 